\title{
Fuentes culturales para el aprendizaje. Estudio en la micro, pequeña y mediana empresa en Celaya
}

\author{
RICARDO CONTRERAS-SOTO ${ }^{\mathrm{a}}$, ALEJANDRA LÓPEZ-SALAZAR ${ }^{\mathrm{b}}$, \\ SALVADOR ESTRADA-RODRÍGUEZ ${ }^{c}$
}

\begin{abstract}
RESUMEN Este trabajo de investigación tiene como objetivo analizar las fuentes de información que utiliza la micro, pequeña y mediana empresa para aprender sobre su actividad empresarial. A través de un análisis cualitativo se entrevistaron a 342 empresas del sector manufacturero de la ciudad de Celaya, Guanajuato, México. Los resultados muestran que las empresas utilizan 26 diferentes fuentes de información para aprender sobre su actividad, las cuales se reagrupan en siete categorías: lógicas interorganizacionales, fuentes especializadas y TICs; fuentes formales de conocimiento; fuentes organizacionales; medios de comunicación y publicidad; fuentes informales de comunicación; experiencia propia del empresario; y ausencia de información y conocimiento. La importancia y uso de cada una de ellas depende del tamaño de la empresa y de la industria a la que pertenece, pero todas ellas contribuyen a desarrollar, consolidar o potenciar vínculos con otras empresas o entes que ayuden a potenciar la capacidad de aprender de la organización.
\end{abstract}

PALABRAS CLAVE conocimiento, fuentes de información, Mipymes, opiniones.

\section{HISTORIA DEL ARTÍCULO}

¿CÓMO CITAR?:

Contreras-Soto, R., López-

Salazar, A. \& Estrada-Rodríguez, S. (2015). Fuentes culturales para el aprendizaje. Estudio en la micro, pequeña y mediana empresa en Celaya. Perspectiva Empresarial, 2(1), 25-44. http:// dx.doi.org/10.16967\%2Frpe. v2n1a2

RECIBIDO: 26 de noviembre de 2013 APROBADO: 5 de junio de 2014

CORRESPONDENCIA:

Salvador Estrada Rodríguez, Av. Ing. Javier Barros Sierra \#201 esq. Av. Baja California; Ejido Santa María del Refugio; Celaya, Gto.; C.P. 38110 México.

a Doctor en Administración por la Universidad de Celaya, profesor-investigador de la Universidad de Guanajuato, Campus Celaya-Salvatierra, México. Correo electrónico: riconsoto@hotmail.com

b Doctora en Negocios y Estudios Económicos por la Universidad de Guadalajara, profesora-investigadora de la Universidad de Guanajuato, Campus Celaya-Salvatierra, México. Correo electrónico: alelopez.salazar@yahoo.com

Doctor en Economía y Gestión de la Innovación y Política Tecnológica por la Universidad Autónoma de Madrid, profesorinvestigador de la Universidad de Guanajuato, Campus Celaya-Salvatierra, México.

Correo electrónico: salvador.estrada@gmail.com 

enterprises in Celaya

ABSTRACT The objective of this research project is to analyze information sources that micro, small and medium-size enterprises use in order to learn about their business activity. 342 enterprises from the manufacturing sector in the city of Celaya, in Guanajuato, Mexico, were interviewed through a qualitative analysis. The results show that these companies use 26 different information sources in order to learn about their activities, which can be re-grouped in seven categories: cross-organizational logic, specialized sources and ICTs; formal sources of knowledge; organizational sources; communication and advertising mediums; informal sources of communication; the entrepreneur's own experience; and the absence of information and knowledge. The importance and use of each of them depends on the size of the enterprise and the type of industry it belongs to; while

¿CÓMO CITO EL ARTÍCULO? HOW TO CITE THIS PAPER?

CHICAGO:

Contreras-Soto, Ricardo, López-Salazar, Alejandra y Estrada-Rodríguez, Salvador. 2015. "Fuentes culturales para el aprendizaje. Estudio en la micro, pequeña y mediana empresa en Celaya". Perspectiva Empresarial 2: 25-44. http://dx.doi. org/10.16967\%2Frpe.v2n1a2

MLA:

Contreras-Soto, Ricardo, LópezSalazar, Alejandra y EstradaRodríguez, Salvador. "Fuentes culturales para el aprendizaje. Estudio en la micro, pequeña y mediana empresa en Celaya". Perspectiva Empresarial 2.1 (2015): 25-44.

Digital. http://dx.doi. org/10.16967\%2Frpe.v2n1a2 they all contribute to developing or promoting bonds with other enterprises or entities that help strengthen the enterprise's learning capability.

KEYWORDS knowledge, information sources, SMEs, opinions.

\section{Fontes culturais para a aprendizagem. Estudo na micro, pequena e média empresa em Celaya}

RESUMO Este trabalho de pesquisa tem como objetivo analisar as fontes de informação que utiliza a micro, pequena e média empresa para aprender sobre sua atividade empresarial. Através de uma análise qualitativa, entrevistou-se a 342 empresas do setor manufatureiro da cidade de Celaya, Guanajuato, México. Os resultados mostram que as empresas utilizam 26 diferentes fontes de informação para aprender sobre sua atividade, as quais se reagrupam em sete categorias: lógicas interorganizacionais, fontes especializadas e TICs; fontes formais de conhecimento; fontes organizacionais; meios de comunicação e publicidade; fontes informais de comunicação; experiência própria do empresário; e ausência de informação e conhecimento. A importância e uso de cada uma delas depende do tamanho da empresa e da indústria a que pertence, mas todas elas contribuem para desenvolver, consolidar ou potenciar vínculos com outras empresas ou entes que ajudem a potenciar a capacidade de aprender da organização.

PALAVRAS CHAVE conhecimento, fontes de informação, Mipymes, opiniões. 


\section{Introducción}

Un sistema de negocios ${ }^{1}$ está conformado por conjuntos de empresas y organizaciones que interactúan competitiva y cooperativamente de acuerdo con su cultura e instituciones. Estas interacciones determinan un potencial competitivo para el territorio que les da asiento productivo y raíz sociocultural. Los factores presentes en el territorio, a su vez, influyen en su desempeño en una causación bidireccional. Cualquier estrategia de desarrollo que busque proveer de ventajas competitivas a sus activos locales debe comprender las configuraciones que estos sistemas empresariales constituyen para potenciar sus vínculos económicos, fortalecer sus relaciones de confian$\mathrm{za}$, aprovechar sus bases de conocimiento y trayectorias de aprendizaje compartido, así como integrar recursos y socios complementarios.

Estas configuraciones se manifiestan a través de canales de comunicación y cooperación donde fluyen recursos, ideas, información, conocimiento, prácticas y creencias. En la nueva economía basada en el conocimiento, el potencial competitivo se revela a través de la dinámica encontrada en estas configuraciones o redes, donde el desempeño superior se alcanza con la participación de numerosas y diversas fuentes de conocimiento pero, también, con la capacidad para utilizarle para los propios fines competitivos, sean estos de mantenimiento, seguimiento, adelantamiento o pionerismo de mercado. Una forma de analizar estas configuraciones es a través del estudio de las fuentes de conocimiento.

El presente trabajo busca explorar, en un territorio determinado, cuáles son las fuentes de información que utilizan los empresarios de las micro, pequeñas y medianas unidades económicas

El sistema de negocios puede definirse como un marco analítico para comparar y contrastar las diferentes maneras en que se organizan las actividades económicas constituidas en diferentes contextos institucionales, en específico de los países (Foss, 1999). En nuestro caso extrapolamos el enfoque para llevarlo al nivel local. Richard Whitley lo define como los arreglos concretos de las relaciones jerarquías-mercados que se han institucionalizado y han tenido relativo éxito en contextos particulares (Whitley, 1992, p. 10). En dichos arreglos se combinan preferencias por ciertas actividades y habilidades con grados diversos de discrecionalidad en la toma de decisiones y en la manera en que se coordinan los actores económicos. manufactureras a fin de analizar las prácticas culturales de su uso.

La importancia del estudio de las fuentes de información para el aprendizaje radica en su aporte explicativo al crecimiento de la empresa y la economía (Romer, 1990). Los flujos entre las empresas y las industrias constituyen un factor endógeno explicativo del crecimiento y competitividad de la economía. Además, estos flujos permiten obtener rendimientos crecientes de las inversiones (Arthur, 1989) y generan efectos positivos, ya que hacen posible la reducción de los riesgos y de la incertidumbre, distribuir mejor la información, compartir y abaratar costos, coordinar la toma de decisiones y asignar los recursos, entre otros.

El desempeño de los agentes económicos está condicionado por la dinámica de estos flujos puesto que el tipo de fuente utilizada determinará, en buena medida, la interacción y los vínculos que logre establecer con el resto de los actores, así como las oportunidades y amenazas que enfrentará (Lugones et al., 2007). Así, los cambios potenciales en los productos y procesos, métodos de gestión y mercados pueden gestarse no solo a través de actividades explícitas de la estrategia de la empresa, sino también por la interrelación con diversas fuentes de conocimiento (Estrada, 2005). En breve, el uso de las fuentes de información condiciona el desarrollo de capacidades competitivas esenciales.

Hillmann (2001, p. 48) hace referencia al aprendizaje como:

[...] los procesos de desarrollo mental, adquisición, ampliación y transformación de conocimientos, capacidad de comprensión, formas de expresión emocional, valores, actitudes, formas de conducta, habilidades y capacidades de resolución de problemas (...) los procesos de aprendizaje se tienen sobre todo en cuenta en las investigaciones sobre: a) la constitución y desarrollo de la sociocultura; b) la integración de personas en grupos, organizaciones e instituciones; c) el desarrollo de orientaciones de valor, actitudes, opiniones, intereses y formas de conducta como resultado de situaciones de comunicación e información social y procesos de conversión de situaciones sociales objetivas en contenidos subjetivos de la conciencia. Sociológicamente, las condiciones históricas, culturales, socioculturales, interpersonales y materiales son especialmente significativas para el aprendizaje. Procesos de aprendizaje en sentido amplio se llevan a cabo, más allá del individuo, también en las formaciones 
sociales y en las sociedades, que lo constituyen una dimensión central del cambio social y, sobre todo, de la evolución sociocultural. En la sociedad moderna, de cambios tan acelerados, que el individuo pueda dominar con éxito su vida y controlar sus posibilidades de futuro depende decisivamente de la disposición y capacidad que tenga de aprender continuamente.

Las fuentes de conocimiento disponibles constituyen un entorno que condiciona el desempeño innovador de una empresa, industria, sector, territorio o país. La literatura económica ha abordado este entorno mediante el estudio de los flujos de conocimientos o derramas ${ }^{2}$ que producen las inversiones extranjeras directas hacia las empresas locales, si bien se han extrapolado desde empresas grandes, sean extranjeras o nacionales (Dutrénit \& De Fuentes, 2009). Por otro lado, la habilidad para hacer un uso eficiente de estas derramas por parte de las pequeñas empresas se ha estudiado a través de las capacidades de absorción, que expresan la habilidad de una empresa en reconocer el valor de información nueva y externa, asimilarla y aplicarla con fines comerciales (Cohen \& Levinthal, 1990). Las capacidades de absorción de las empresas reflejan sus bases de conocimiento, entendidas como información, conocimiento y capacidades para buscar soluciones innovadoras (Dosi, 1982, 1988 y 1997). Esto implica también un proceso de aprendizaje. Un enfoque similar presenta la teoría proveedor-usuario ${ }^{3}$, derivada de la aproximación relacional al estudio de la innovación (Lundvall, 1992), donde el grado de las vinculaciones, el intercambio de información y

2 Las derramas son transferencias de conocimiento que resultan en incrementos de la productividad del agente que las recibe, o bien en facilidad para ingresar a un nuevo mercado (Blomström y Kokko, 1998). La primera ocurre cuando "la entrada o la presencia de filiales de las empresas multinacionales producen beneficios de productividad o eficiencia en las empresas locales y las multinacionales no son capaces de internalizar el valor total de estos beneficios". Las derramas de acceso al mercado ocurren cuando la actividad exportadora de una multinacional reduce el costo de comenzar a exportar de las empresas nacionales (Aitken et al., 1997).

3 Se entiende como relación proveedor-usuario las vinculaciones comerciales directas o indirectas entre entidades independientes en las que el usuario compra los productos del proveedor y los incorpora a su proceso productivo (Hernández, 1998). los lazos de cooperación constituyen el objeto de estudio.

Los modelos y marcos analíticos desarrollados para el estudio de la innovación señalan que la naturaleza y los resultados del esfuerzo innovador dependen en gran medida de las fuentes de información y conocimiento. Así, el Manual de Oslo (OCDE, 2005, p. 88) establece que:

Las actividades de innovación dependen en parte de la diversidad y estructura de los vínculos con las fuentes de información (...) Los vínculos actúan como fuentes de conocimiento y tecnología para la actividad innovadora de la empresa, abarcando desde las fuentes pasivas de información hasta los proveedores de conocimiento y tecnología -incorporada o no- pasando por los consorcios de cooperación.

La literatura señala que son diversas las fuentes o mecanismos por los que se producen las derramas, entre las que se destaca la movilidad del capital humano y los vínculos de proveeduría (Duténit \& De Fuentes, 2009). Cohen \& Levinthal (1990) mencionan que la capacidad de absorción puede ser generada y fortalecida a través de diversos medios: 1) como un subproducto de la I+D; 2) como un subproducto de las operaciones de manufactura de la empresa, y 3) por inversión directa, mediante la capacitación o contratación del personal. Por su parte, las encuestas de innovación revelan el predominio y la importancia de los distintos tipos de vínculos y los factores que influyen en su utilización. Un estudio en los países de la OCDE (2000) muestra que las fuentes de innovación incluyen la cooperación con otras empresas por medio de redes, alianzas y joint-ventures; compra de equipamientos, involucramiento con servicios especializados en uso intensivo de conocimiento, interacción con instituciones científicas, integración de empresas por medio de fusiones y adquisiciones, así como movilidad del capital humano altamente cualificado.

En el presente estudio se abordan las diversas fuentes de información para el aprendizaje, indagando en los sujetos de estudio la propia experiencia productiva, de tal suerte que sean los mismos entrevistados quienes descubran dichas fuentes y valoricen su utilización.

En conclusión, ¿cuáles son las fuentes culturales de información que utilizan las empresas para aprender sobre su actividad? Esta es la pregunta que guía el presente estudio. 


\section{Método}

El método se enfocó desde una perspectiva cualitativa de exploración hermenéutica en la interpretación de la doxa ${ }^{4}$ de las entrevistas y en la aproximación de la contextualización organizacional, tomando como eje la pregunta abierta: ¿Qué otras fuentes conoce o utiliza para aprender a conocer sobre su actividad empresarial?

El levantamiento de la información se registró de forma escrita y grabada. Se transcribió en un archivo procesador de palabras Word, donde se le asignó un número a la entrevista y se archivó en una carpeta. Se procedió a estructurar una base de datos en Excel de manera matricial, en la cual las preguntas se ubicaron verticalmente mientras que los casos se colocaron horizontalmente.

\section{Procedimiento de análisis}

Para desarrollar la clasificación de las fuentes de información se partió de la evidencia discursiva de los testimonios para operativamente acercarse a la estructuración teórica conceptual, utilizando los criterios básicos que las personas referenciaban. De esta forma se encontraron en el discurso
28 categorías, una de las cuales explicita el no conocimiento de las fuentes y otra el hecho de no utilizar ninguna. Esto, en relación con el análisis cualitativo. En lo relativo al análisis cualitativo, se utilizaron estadísticas descriptivas.

\section{Descripción de la fuente de información}

De acuerdo con los datos del Instituto Nacional de Estadística y Geografía (Inegi), de México (2010), se informa que en el municipio de Celaya existen 1.707 unidades económicas, las cuales conforman nuestro universo de análisis. El cálculo de la muestra se hizo con el programa Decision Analyst STATS considerando un margen de error del 5\%, una probabilidad de selección del $50 \%$ y un nivel de confianza del $95 \%$. Estos parámetros de diseño arrojaron una muestra de 314 empresas, la cual representa el 18\% del universo. Durante el trabajo de campo se aplicó el cuestionario a 342 establecimientos realizando la entrevista a los dueños o responsables de los mismos, con lo cual se obtuvo una representatividad del $20 \%$.

TABLA 1. Distribución sectorial y por tamaño de la muestra general

\begin{tabular}{|c|c|c|c|c|c|c|}
\hline \multirow[b]{2}{*}{ GIRO } & \multicolumn{5}{|c|}{ TAMAÑO DE LA EMPRESA } & \multirow[b]{2}{*}{ Total } \\
\hline & $\mathrm{Nc}$ & Microempresa & $\begin{array}{l}\text { Pequeña } \\
\text { empresa }\end{array}$ & $\begin{array}{l}\text { Mediana } \\
\text { empresa }\end{array}$ & $\begin{array}{l}\text { Gran } \\
\text { empresa }\end{array}$ & \\
\hline Agricultura y ganadería & 0 & 4 & 4 & 1 & 0 & 9 \\
\hline Construcción & 0 & 15 & 6 & 1 & 1 & 23 \\
\hline Industria de alimentos & 1 & 139 & 25 & 4 & 1 & 170 \\
\hline Industria de bebidas & 0 & 6 & 1 & 0 & 0 & 7 \\
\hline Industria textil & 0 & 15 & 4 & 1 & 1 & 21 \\
\hline Industria química & 0 & 3 & 0 & 1 & 0 & 4 \\
\hline Industria plástica & 0 & 2 & 3 & 0 & 2 & 7 \\
\hline Industria mineral & 0 & 3 & 3 & 0 & 0 & 6 \\
\hline Industria metálica & 1 & 3 & 3 & 0 & 0 & 7 \\
\hline Industria de muebles & 1 & 7 & 0 & 0 & 0 & 8 \\
\hline Comercio & 1 & 16 & 6 & 0 & 0 & 23 \\
\hline Servicios & 0 & 36 & 6 & 0 & 0 & 42 \\
\hline NC & 0 & 9 & 4 & 2 & 0 & 15 \\
\hline TOTAL & 4 & 258 & 65 & 10 & 5 & 342 \\
\hline
\end{tabular}

Fuente: elaboración propia

4 Se refiere a la opinión o conocimiento empírico. 


\section{Muestra con referencia al tamaño}

De las 342 entrevistas realizadas, 258 son microempresas que representan el $75,43 \%$ de la muestra; el número de pequeñas fue de 65 y representan el 19\%; las obtenidas en medianas empresas fueron diez y representan el 2,9\%; y, por último, cinco empresas son grandes y representan el $1,46 \%$ de la muestra. Cuatro empresas omitieron el personal que trabajan con ellas, y por tanto no se pudo determinar su tamaño.

\section{Muestra con referencia al giro}

La muestra se concentra en el sector manufacturero, el cual tiene una participación del 67\%, seguido del sector de los servicios que participa con el $12 \%$ de las unidades de producción encuestadas, mientras que la construcción y el comercio representan el $7 \%$ de la muestra cada uno, y finalmente, el sector agropecuario agrupa el 3\% de las unidades de observación. No hubo respuesta en $4 \%$ de los establecimientos visitados.

\section{Descripción del estudio}

México experimenta una transformación en su estructura productiva donde los servicios ocupan una posición preponderante, mientras que, con ciertas fluctuaciones, las manufacturas inician una tendencia decreciente, representando $25,8 \%$ del PIB en 2003. Esto refleja un proceso de reestructuración territorial donde los estados pueden estar mudando o reforzando su estructura productiva. Para el caso de Guanajuato, a raíz de una serie de inversiones productivas desde los años 1970 y 1980, se está reforzando su proceso de industrialización (Garza, 2009), y mantiene un sector terciario pujante que se ha desarrollado de la mano con su dinámica poblacional (Estrada, Cárcamo \& Martín, 2013). Esta entidad federativa presenta un patrón de desarrollo policéntrico que se manifiesta en sus tres zonas metropolitanas y en sus dos ciudades más importantes.

La Zona Metropolitana de Celaya, compuesta por los municipios de Celaya, Villagrán y Cortázar, es la segunda en importancia en el estado, solo detrás de la de León. Se encuentra en el corredor industrial del Bajío donde se concentra la actividad secundaria.

En Celaya la importancia de las manufacturas ha ido ganando terreno pues su participación excede el 50\% del PIB municipal. Las ramas más destacadas están encabezadas por la de electrodomésticos (13,4\%), seguida por la de hierro y acero $(9,2 \%)$ y autopartes $(7,5 \%)$; las dos primeras concentran la totalidad de la actividad en el Estado de Guanajuato, en tanto que las autopartes representan el $40 \%$ del total estatal. Otra media docena de ramas vinculadas a alimentos (principalmente de comercialización, así como de conservación de alimentos), productos químicos y de plástico también presentan cierta relevancia. En Celaya, como en otras concentraciones urbanas, se desarrollan con buen ritmo las actividades de edificación, infraestructura, restaurantes y hoteles, servicios financieros, educativos, de transporte y la mayoría de los comercios (Unger, 2009).

La dinámica de estas ramas sugiere un patrón de especialización municipal basado en economías de aglomeración y plantas de gran escala, principalmente en manufacturas. Por ejemplo, en la rama de electrodomésticos destaca la importancia de la planta de Mabe, con presencia, incluso, en mercados de exportación. En la de hierro y acero es notable la empresa Siderúrgica del Bajío S.A. de C.V., además de otro grupo de empresas concentradas en las autopartes y químicos que son dominantes en el clúster de Celaya, cuya dinámica parece estar influida por la proximidad con la ciudad industrial de Querétaro (Unger, 2009).

\section{Desarrollo}

El análisis exploratorio revela que los empresarios declararon utilizar 26 fuentes diferentes para aprender sobre su actividad empresarial. Estas respuestas fueron establecidas en el $83 \%$ de los casos estudiados. Sin embargo, se destaca que el 13\% de los entrevistados afirma que no utiliza ninguna fuente de información para el aprendizaje. La distribución de las respuestas sobre los recursos de información empleados por los empresarios se muestra en la tabla 2.

Las modalidades identificadas con mayor preponderancia como fuente de información fueron el internet y las revistas (por al menos 17\% de los entrevistados). También destacan las fuentes derivadas de la experiencia productiva de otras empresas y de la propia, con un $12 \%$. Un tercer grupo de fuentes está conformado por los cursos, libros, ferias y catálogos, que fueron señalados por entre el 6 y el $9 \%$ de los entrevistados. Finalmente, se tiene a los proveedores y familiares como 
TABLA 2. Fuentes de información para el aprendizaje de empresarios celayenses de Mipymes

\begin{tabular}{|c|c|c|c|c|}
\hline \multirow{2}{*}{$\begin{array}{l}\text { FUENTES DE INFORMACIÓN PARA } \\
\text { EL APRENDIZAJE }\end{array}$} & \multicolumn{3}{|c|}{ TAMAÑO DE LA EMPRESA } & \multirow{2}{*}{ TOTAL } \\
\hline & Microempresa & Pequeña empresa & Mediana empresa & \\
\hline Internet & $22,86 \%$ & $40,00 \%$ & $20,00 \%$ & $26,31 \%$ \\
\hline Revistas & $15,89 \%$ & $18,46 \%$ & $40,00 \%$ & $17,25 \%$ \\
\hline Experiencias de otras empresas & $12,00 \%$ & $12,30 \%$ & $20,00 \%$ & $11,98 \%$ \\
\hline Experiencia directa & $12,40 \%$ & $12,30 \%$ & $0,00 \%$ & $11,69 \%$ \\
\hline Cursos & $8,91 \%$ & $6,15 \%$ & $20,00 \%$ & $9,06 \%$ \\
\hline Libros & $6,59 \%$ & $9,23 \%$ & $10,00 \%$ & $7,01 \%$ \\
\hline Ferias & $5,81 \%$ & $7,69 \%$ & $30,00 \%$ & $6,72 \%$ \\
\hline Catálogos & $6,20 \%$ & $6,15 \%$ & $10,00 \%$ & $6,14 \%$ \\
\hline Proveedores & $5,03 \%$ & $7,69 \%$ & $0,00 \%$ & $5,55 \%$ \\
\hline Familiares & $3,10 \%$ & $4,61 \%$ & $0,00 \%$ & $3,50 \%$ \\
\hline Consulta de gobierno & $1,16 \%$ & $3,07 \%$ & $20,00 \%$ & $2,33 \%$ \\
\hline Seminarios & $1,93 \%$ & $1,53 \%$ & $20,00 \%$ & $2,33 \%$ \\
\hline Televisión & $1,93 \%$ & $3,07 \%$ & $0,00 \%$ & $2,04 \%$ \\
\hline Periódicos & $2,32 \%$ & $1,53 \%$ & $0,00 \%$ & $2,04 \%$ \\
\hline Capacitaciones & $1,93 \%$ & $1,53 \%$ & $0,00 \%$ & $1,75 \%$ \\
\hline Consulta a instituciones & $1,16 \%$ & $3,07 \%$ & $10,00 \%$ & $1,75 \%$ \\
\hline Cámaras empresariales & $1,16 \%$ & $3,07 \%$ & $0,00 \%$ & $1,75 \%$ \\
\hline Estudios & $0,77 \%$ & $3,07 \%$ & $0,00 \%$ & $1,46 \%$ \\
\hline Conferencias & $0,77 \%$ & $4,61 \%$ & $0,00 \%$ & $1,46 \%$ \\
\hline Publicidad & $1,55 \%$ & $0,00 \%$ & $0,00 \%$ & $1,17 \%$ \\
\hline Experiencia en otros trabajos & $0,77 \%$ & $3,07 \%$ & $0,00 \%$ & $1,17 \%$ \\
\hline Certificaciones & $0,38 \%$ & $1,53 \%$ & $0,00 \%$ & $1,17 \%$ \\
\hline Talleres & $0,77 \%$ & $0,00 \%$ & $0,00 \%$ & $0,58 \%$ \\
\hline Asesorías & 0 & $1,53 \%$ & $10,00 \%$ & $0,58 \%$ \\
\hline Reuniones o juntas & $0,77 \%$ & $0,00 \%$ & $0,00 \%$ & $0,58 \%$ \\
\hline Consulta a clientes & $0,38 \%$ & $0,00 \%$ & $0,00 \%$ & $0,29 \%$ \\
\hline No sabe & $3,10 \%$ & $0,00 \%$ & $0,00 \%$ & $2,33 \%$ \\
\hline Ninguna & $13,95 \%$ & $9,23 \%$ & $0,00 \%$ & $12,86 \%$ \\
\hline
\end{tabular}

Fuente: elaboración propia

principales fuentes de conocimiento, los cuales representan entre el 4 y el $6 \%$.

Otro conjunto de 16 modalidades de fuentes resulta conocido o utilizado, aunque en un porcentaje muy bajo. Es una mezcla diversa donde hay fuentes de acceso libre, catalogadas, con componentes tácitos y relacionales. Al parecer, su naturaleza no es muy diferente a la mezcla encontrada en las fuentes más utilizadas o conocidas.

En cuanto a diferencias por tamaño, los datos están dispersos, por lo que puede suponerse una influencia en el uso de la información dependiendo de la talla del establecimiento. Al hacer un test de correlación de rangos se encuentra una correlación media entre las micro y las pequeñas empresas, pero baja con respecto al estrato inmediato superior de las medianas empresas ${ }^{5}$. En este segmento se encuentran algunas peculiaridades como un porcentaje mayor de usuarios o conocedores de las ferias y revistas con respecto

5 Los coeficientes de correlación entre las categorías de conocimiento o uso de fuentes de información entre los micro y pequeños empresarios fue de 0,78 , entre los pequeños $y$ medianos, 0,48, y entre los micro y medianos, 0,43. 
al internet, o del acceso o consulta al gobierno, seminarios, instituciones o asesorías en detrimento de la propia experiencia, los proveedores y las familias.

Al comparar estos datos con los estudios empíricos encontramos que, en otras latitudes, el enfoque ha sido el atribuir a estas fuentes una influencia sobre la capacidad de innovación, mientras que nuestro enfoque estaría más orientado al desarrollo de una capacidad empresarial general, con competencias más básicas, pero asumiendo que podrían llegar a detonar un potencial innovador -para este contexto socio-territorial y cultural más acotado-.

Se hizo una revisión no exhaustiva de 22 estudios sobre fuentes de información en estudios empíricos (tabla 3 y anexo I). A diferencia de nuestro estudio, sólo en un tercio de ellos resultaron muy importantes las fuentes catalogadas -en las que incluimos junto con las publicaciones al internet-; sin embargo, para los estudios encontrados en México y además los que consideran a las pymes, la importancia aumenta.

En cuanto a las fuentes basadas en experiencias propias, dos tercios encuentran que tienen una importancia capital, tendencia que se mantiene tanto en los estudios de países desarrollados como en los latinoamericanos. Para el caso mexicano, algunos estudios no indagan sobre esta fuente, por lo que parece que tuvieran menos importancia; sin embargo, para los estudios hechos en profundidad, y en particular para los relativos a las capacidades de aprendizaje e innovación, las experiencias propias y las fuentes internas resultan ser los canales más importantes. Así, la evidencia apunta a que las fuentes de información tecnológica e innovación más importantes para las empresas mexicanas están dentro de ellas mismas (Arvanitis \& Villavicencio, 1998; LópezLeyva, 2003). Además, es notable que el desarrollo y la modificación de productos y procesos se hace, mayoritariamente, por cuenta propia. Los departamentos de producción, seguidos por el de mercadotecnia e ingeniería, son valorados por las empresas innovadoras como las fuentes de información significativamente más importantes (Conacyt, 1999 y 2003). Existe evidencia de que las empresas manufactureras, en general, prefieren las fuentes internas a las externas, y que consideran la experiencia, el conocimiento y las

TABLA 3. Síntesis de la evidencia encontrada en estudios empíricos (22 casos)

\begin{tabular}{|c|c|c|c|c|c|c|c|c|c|c|c|c|}
\hline $\begin{array}{l}\text { FUENTES DE INFORMACIÓN } \\
\text { Y CONOCIMIENTO }\end{array}$ & $\frac{\overrightarrow{1}}{\circ}$ & 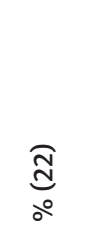 & 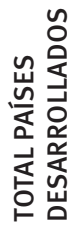 & $\underbrace{\overparen{0}}_{0}$ & 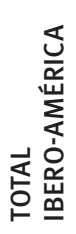 & $\underset{0}{\stackrel{\Xi}{E}}$ & 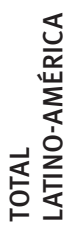 & $\underset{0}{\stackrel{\overbrace{}}{E}}$ & 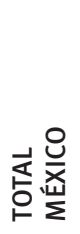 & $\underbrace{\sigma}_{0}$ & 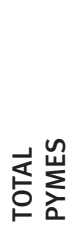 & 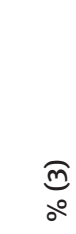 \\
\hline Revistas especializadas & 4 & $18 \%$ & 2 & $33 \%$ & 3 & $18 \%$ & 2 & $13 \%$ & 2 & $22 \%$ & 1 & $33 \%$ \\
\hline Libros & 1 & $5 \%$ & 0 & $0 \%$ & 1 & $6 \%$ & 1 & $7 \%$ & 1 & $11 \%$ & 1 & $33 \%$ \\
\hline Ferias y exposiciones & 7 & $32 \%$ & 2 & $33 \%$ & 6 & $35 \%$ & 5 & $33 \%$ & 4 & $44 \%$ & 0 & $0 \%$ \\
\hline Competidores & 6 & $27 \%$ & 1 & $17 \%$ & 6 & $35 \%$ & 5 & $33 \%$ & 3 & $33 \%$ & 0 & $0 \%$ \\
\hline Grupos internos & 14 & $64 \%$ & 4 & $67 \%$ & 12 & $71 \%$ & 10 & $67 \%$ & 5 & $56 \%$ & 1 & $33 \%$ \\
\hline Clientes & 19 & $86 \%$ & 5 & $83 \%$ & 16 & $94 \%$ & 14 & $93 \%$ & 8 & $89 \%$ & 3 & $100 \%$ \\
\hline Univ. y Centros I+D & 4 & $18 \%$ & 3 & $50 \%$ & 3 & $18 \%$ & 1 & $7 \%$ & 0 & $0 \%$ & 0 & $0 \%$ \\
\hline Gobierno & 1 & $5 \%$ & 1 & $17 \%$ & 0 & $0 \%$ & 0 & $0 \%$ & 0 & $0 \%$ & 1 & $33 \%$ \\
\hline Capacitación & 2 & $9 \%$ & 1 & $17 \%$ & 1 & $6 \%$ & 1 & $7 \%$ & 0 & $0 \%$ & 0 & $0 \%$ \\
\hline Conferencias y simposios & 2 & $9 \%$ & 0 & $0 \%$ & 2 & $12 \%$ & 2 & $13 \%$ & 1 & $11 \%$ & 0 & $0 \%$ \\
\hline Internet & 2 & $9 \%$ & 0 & $0 \%$ & 2 & $12 \%$ & 2 & $13 \%$ & 2 & $22 \%$ & 2 & $67 \%$ \\
\hline Consultores & 6 & $27 \%$ & 1 & $17 \%$ & 6 & $35 \%$ & 5 & $33 \%$ & 3 & $33 \%$ & 1 & $33 \%$ \\
\hline Patentes & 0 & $0 \%$ & 0 & $0 \%$ & 0 & $0 \%$ & 0 & $0 \%$ & 0 & $0 \%$ & 0 & $0 \%$ \\
\hline
\end{tabular}


habilidades de sus ingenieros, técnicos y obreros como la fuente más relevante de innovación (Corona \& Hernández, 2000). Estas son fuentes culturales de aprendizaje.

La fuente constituida por alguna forma de capacitación y también las ferias resultaron importantes para la mitad de los estudios revisados. En México se hace énfasis un poco más en esta fuente. Los estudios sobre pymes que revisamos no nos permiten ratificar nuestros hallazgos, dado que no revelan esta información. Por otro lado, cabe destacar que las ferias y exposiciones tienen mayor relevancia en los ámbitos ibero y latinoamericano, que entre los países desarrollados donde hay un mayor interés hacia las universidades y los centros de investigación.

Nuestra revisión también muestra que los clientes constituyen la fuente de información más importante, seguida de los proveedores. Esta situación contrasta fuertemente con nuestra evidencia, pues si bien los proveedores son mencionados con mayor preponderancia, los clientes son reconocidos solo por tres de cada mil. Es posible que esta distorsión se deba a una interpretación de nuestros entrevistados en cuanto a las fuentes de información utilizadas para mejorar la capacidad empresarial propia.

En cuanto a que exista alguna diferencia en la valoración influida por el tamaño, Barañano (1998) y Quadros et al. (2001) encuentran alguna evidencia que podría soportar nuestros resultados. En general, refieren que las fuentes de información menos formalizadas son más accesibles y asequibles a las pymes. Los estudios específicos que solo consideran las pymes también apuntan hacia esta dirección con fuentes catalogadas de libre acceso además de la propia experiencia (CIPI, 2003; Ollivier \& Thompson, 2009).

De esta revisión se podría concluir, preliminarmente, que las empresas establecen ciertos patrones regulares en cuanto a sus fuentes de adquisición de información para el aprendizaje de su actividad, siendo muy importantes los clientes, proveedores y la propia experiencia productiva (incluido el trabajo de sus grupos internos y trabajadores), añadiendo, para el ámbito iberoamericano, las ferias y exposiciones, y considerando para las pymes, además, las fuentes catalogadas.

Nuestros resultados permiten inferir que para nuestro caso de Mipymes en Celaya, las principales fuentes de aprendizaje son los contactos personales, los proveedores de cursos, las ferias, los proveedores de insumos y las familias. Las fuentes externas principales serían las de libre acceso como internet, revistas, libros y catálogos. En cuanto a las fuentes internas, la principal sería la propia experiencia productiva.

\section{Valoración cualitativa}

Para lograr el objetivo del estudio no basta con hacer una referencia de fuentes; es necesario analizarlas con referencia a las prácticas culturales del uso de dichas fuentes por los empresarios. Entonces optamos por aglutinar las fuentes de acuerdo con sus fines, tomando en cuenta las prácticas culturales en relación con las consultas de dichas fuentes, sus implicaciones, el grado de complejidad social (en cuanto al uso de su codificación para el conocimiento y socialización en sus campos), su disponibilidad, confiabilidad y consistencia.

Se realizaron varios intentos para distinguir y agrupar la importancia del manejo de determinadas fuentes de información y conocimiento con referencia a otras. En los intentos se estableció un criterio lógico ordinal, para ir de las fuentes más adecuadas y confiables a las menos confiables, y también de agruparlas como excelentes, buenas, regulares, bajas, malas y ausentes. Era lo más operativo, pero muchos de estos criterios corren el riesgo de desdeñar los procesos complejos culturales que realmente se dan, en este caso, en la periferia, y que ante esta lectura es "desorden" o "malformación" de los empresarios; donde, por tanto, el orden, es el orden del poder, el deber ser, lo dan la "modernidad" y la "evolución" de las fuentes, aunado a las inducciones que hacen las sociedades "desarrolladas".

Este tipo de evaluación no deja pensar en las grandes desigualdades en cuanto al capital cultural de los actores de la sociedad, la complejidad como se estructuran las ideas más como recursos de distintas fuentes (ciencia, doxa, religión, desconocimiento, empírea, etcétera) y perspectivas entretejidas en una especie de "bricolaje" ${ }^{6}$, donde los conocimientos e ideas son de procedencia (en las distintas fuentes y en distintas configuraciones) de los empresarios latinoamericanos; en el caso

6 Concepto creado por Claude Lévi-Strauss para tratar de formular cómo reconstruye las ideas de las cosas, sus efectos simbólicos en las reinterpretaciones que se dan en distintas manifestaciones de las prácticas culturales. 
de México, referidos a las micro, pequeñas y medianas empresas, como operan.

Por otra parte, la dirección de la cuestión ordinal hace parecer que unos medios son mejores que otros, o que la idea es mejorar los criterios inferiores por unos superiores (cuando de hecho se pueden dar simultáneos). Por ello, se agruparon según los fines de las fuentes de información en que fueron diseñados, es decir, el uso adecuado de los recursos de información (pero a sabiendas de que su diversidad no era una cuestión normativa). Entonces se replantea su valor cultural de uso y disponibilidad apoyados en la analogía ${ }^{7}$ (ver anexo II), quedando así la propuesta tentativa distribuida en siete grupos:

1. Lógicas interorganizacionales, fuentes especializadas y TIC: se agruparon aquí las lógicas interorganizacionales en donde la información en un campo determinado muestra y comparte el nivel de práctica objetivada en sus productos. También se dan las fuentes especializadas que permiten conocer específicamente cuestiones sobre productos o servicios, como son revistas y catálogos, la generación de conocimiento dada por la investigación directa (en este caso, por la consulta a instituciones), los aprendizajes adquiridos en las experiencias en las ferias, e internet como medio por excelencia de las TIC.

2. Fuentes formales de conocimiento: son aquellas maneras en que se han institucionalizado las formas de aprender (de manera formal). En este caso, emitidas por instituciones, consultores, instituciones públicas o personas que tengan el conocimiento técnico.

3. Fuentes organizacionales: son aquellas fuentes que como eje están determinadas por organizaciones públicas y privadas que se han construido partiendo de convenciones en el campo propio.

4. Medios de comunicación y publicidad: son aquellos medios de comunicación más

7 La estrategia del planteamiento es reconsiderar el problema de cómo se configuran las ideas ante realidades distantes y ante recursos y acervos disponibles, impulsados en una especie de amalgamas de distintas configuraciones. La estrategia es ver ese orden simbólico distinto, que puede ayudar a detonar de acuerdo con sus condiciones y sus posibilidades. comunes, como la radio, la televisión y los diarios. Por otro lado, son las fuentes que como fin tienen vender y están diseñadas para persuadir la compra de bienes o servicios.

5. Otras formas culturales de información: hace referencia a las formas informales de comunicación que se dan entre grupos de una cultura determinada, en este caso la comunicación que existe entre parientes en relación con negocios.

6. No relación con otras fuentes: se refiere a los casos en los que no existe más fuente de conocimiento que la práctica inmediata del empresario como agente referente.

7. Ausencia de información y conocimiento: son el desconocimiento o ausencia de fuentes.

Es importante señalar que de acuerdo con los testimonios de los empresarios no se utilizaron otras fuentes, y esto es grave porque manifiesta la ausencia de innovación, como es la consulta de patentes, de observatorios, de artículos en específico de internet (o portales de información pertinente) sobre procesos productivos o materiales.

Una vez establecidos los criterios de aglutinación, se analiza su ponderación (tabla 4).

La reflexión realizada indica que cerca del $12 \%$ de las empresas utilizan lógicas interorganizacionales, fuentes especializadas y TIC como fuentes de información para aprender su actividad. En este rubro, las revistas e internet son las principales fuentes de información tanto para la micro y pequeña como para la mediana empresa. El aspecto amigable y didáctico del internet ha permitido ampliar la visión de lo social (la geografía), dado que se consultan productos y mercados nacionales e internacionales. El uso de internet de manera coordinada puede impulsar la inteligencia competitiva de la empresa. Existe una clara diferencia con la mediana empresa en relación con la asistencia a ferias y exposiciones, pues una tercera parte de ellas utiliza esta fuente de información. En lo asociado con la consulta a instituciones, es importante puesto que refleja el grado de vinculación entre institutos de educación superior o centros de investigación y empresas. Aunque es muy baja la relación entre el número de empresas y la vinculación de investigación, las pocas empresas que lo hacen, consultan a los centros tecnológicos y a las universidades en cuestiones técnicas específicas. 
TABLA 4. Principales fuentes de información para el aprendizaje por tamaño de empresa

\begin{tabular}{|c|c|c|c|c|c|}
\hline \multicolumn{3}{|c|}{ FUENTES DE INFORMACIÓN PARA EL APRENDIZAJE } & \multicolumn{2}{|l|}{ TAMAÑO } & \multirow[b]{2}{*}{ Promedio genera } \\
\hline General & Particular & Micro & Pequeña & Mediana & \\
\hline \multicolumn{2}{|c|}{$\begin{array}{l}\text { Lógicas interorganizacionales, fuentes } \\
\text { especializadas y TIC }\end{array}$} & $10,38 \%$ & $15,07 \%$ & $22 \%$ & $11,63 \%$ \\
\hline & Consulta a instituciones & $1 \%$ & $3 \%$ & $10 \%$ & \\
\hline & Revistas & $15,89 \%$ & $18,46 \%$ & $40 \%$ & \\
\hline & Ferias y exposiciones & $6 \%$ & $7,5 \%$ & $33,3 \%$ & \\
\hline & Catálogos & $6 \%$ & $6 \%$ & $10 \%$ & \\
\hline & Internet & $23 \%$ & $40 \%$ & $20 \%$ & \\
\hline \multicolumn{2}{|c|}{ Fuentes formales de conocimiento } & $2,7 \%$ & $3,46 \%$ & $7,5 \%$ & $3,02 \%$ \\
\hline & Talleres & $1 \%$ & & & \\
\hline & Asesorías & & $1,5 \%$ & $10 \%$ & \\
\hline & Cursos & $1 \%$ & $6 \%$ & $10 \%$ & \\
\hline & Libros & $7 \%$ & $9 \%$ & $10 \%$ & \\
\hline & Seminarios & $2 \%$ & $1,5 \%$ & $20 \%$ & \\
\hline & Capacitaciones & $2 \%$ & $2 \%$ & & \\
\hline & Estudios & $1 \%$ & $3 \%$ & & \\
\hline & Conferencias & $1 \%$ & $4,5 \%$ & & \\
\hline \multicolumn{2}{|c|}{ Fuentes organizacionales } & $2,70 \%$ & $3,84 \%$ & $5 \%$ & $3,10 \%$ \\
\hline & Experiencias de otras empresas & $12 \%$ & $12 \%$ & $20 \%$ & \\
\hline \multicolumn{6}{|c|}{ Consulta a clientes } \\
\hline & Proveedores & $5 \%$ & $7,5 \%$ & & \\
\hline & Consulta a gobierno & $1 \%$ & $3 \%$ & $2 \%$ & \\
\hline & Cámaras empresariales & $1 \%$ & $3 \%$ & & \\
\hline & Certificaciones & & $1,5 \%$ & & \\
\hline & Experiencia en otros trabajos & $1 \%$ & $3 \%$ & & \\
\hline & Reuniones o juntas & $1 \%$ & & & \\
\hline Medios d & municación y publicidad & $1,93 \%$ & $1,53 \%$ & ०\% & $1,95 \%$ \\
\hline & Televisión & $2 \%$ & $3 \%$ & & \\
\hline & Periódicos & $1,5 \%$ & $2 \%$ & & \\
\hline & Publicidad & $1,5 \%$ & & & \\
\hline Otras forr & culturales de información & $3,10 \%$ & $4,61 \%$ & $0 \%$ & $3,50 \%$ \\
\hline & Familiares & $3 \%$ & $4,5 \%$ & & \\
\hline No relaci & on otras fuentes & $12,30 \%$ & $11,69 \%$ & $0 \%$ & $11,69 \%$ \\
\hline & Experiencia directa & $12 \%$ & $12 \%$ & & \\
\hline Ausencia & información y conocimiento & $8,52 \%$ & $4,61 \%$ & $0 \%$ & $7,60 \%$ \\
\hline & No sabe & $3,1 \%$ & & & \\
\hline & Ninguna & $13,95 \%$ & $9,23 \%$ & & \\
\hline
\end{tabular}


Solo el 3\% de las empresas utilizan fuentes formales de información. La principal fuente utilizada por la microempresa son los libros, seguida de seminarios y capacitaciones (desarrollo de destrezas). La microempresa no tiene el hábito de asesorarse con un especialista que le permita dar una opinión calificada; suponemos que se debe a sus condiciones económicas. En la pequeña empresa los libros, los cursos y las conferencias son las principales fuentes de información como base del aprendizaje. Y en la mediana empresa se destacan los seminarios, principalmente, aunque utilizan también asesorías (contratación de expertos), cursos y libros, en mayor medida que la micro y la pequeña empresa. Los cursos los ofrecen algunos proveedores, agentes libres y el gobierno para pequeños y microempresarios.

El uso de fuentes organizacionales para la obtención de conocimiento e información representa el 3\% de la muestra. Una de las formas más interesantes de aprendizaje es a través de las experiencias de otras empresas. Existen testimonios que se basan en su capital social, dado por la confianza y por las buenas relaciones; por ejemplo, visitando a otras empresas, a personas del ramo que se apoyan (como buen gremio), recibiendo consejos, apoyo y correspondencia con otros productores, cooperación productiva, intercambiando ideas o con información en la cadena de productiva. Mientras que otras se dieron por una especie de espionaje (copiando, dicen ellos) de sus procesos o sus productos. Esta forma de aprendizaje es mayor en la mediana empresa (20\%) comparada con la utilizada en la micro y pequeña empresa (12\%). La opinión directa del cliente se ve como un gesto de atención o de preocupación; sin embargo el problema es que dificilmente las personas van a hablar mal del producto con el dueño (si lo pueden hacer). Se considera que lo correcto es hacerlo de manera sistemática, no solo como gesto de atención (aunque sabemos que le da calor humano preguntar directamente), pero se considera importante saber qué piensan los consumidores, para que tenga efecto en el producto o servicio o en la innovación de estos. Los casos se presentan solo en las microempresas. A través de los proveedores se entreteje el capital social, y con ello se construye el mercado social. Según los testimonios, existe un buen acercamiento a los clientes por parte de los proveedores, se genera confianza y constantemente se apoyan y dan información. Esta forma de relación existe en el 5\% de las microempresas y entre el 7 y $8 \%$ en las pequeñas.
La tendencia a consultar programas específicos de orientación a las empresas, la participación en distintas cámaras empresariales, las juntas y las certificaciones presenta niveles muy bajos, independientemente del tamaño de la empresa. Pocas empresas consideran su experiencia en otros trabajos como fuente de información para aprender sobre las actividades de su actual empresa.

Los medios de comunicación y publicidad apenas son utilizados como fuentes de conocimiento en menos del 2\% de las empresas. Estos medios incluyen la televisión, los periódicos y la publicidad.

En cuanto a las otras formas de información, cabe señalar la importancia de la familia en la cultura como cadena de transmisión del conocimiento ${ }^{8}$. Estos resultados nos dicen que la información no solo fue para la formación, sino que es constante, frecuente, viva y está siempre disponible, ya que en los miembros de la familia existe la confianza y se puede consultar con base en su experiencia.

La experiencia directa es fundamental, pero es también un discurso autorreferido para justificar el aislamiento de la empresa, que nosotros hemos denominado "el laberinto de la soledad". En la micro y pequeña empresa es donde sucede esta forma de actuar en la cultura empresarial, con un $12 \%$.

En cuanto a la ausencia de información y conocimiento, de cada 100 personas aproximadamente la sexta parte no sabe nada de fuentes, principalmente en las microempresas.

En resumen, se puede decir:

- Considerar la práctica del empresario como fuente única de aprendizaje resulta la más importante por su representación relativa con un $11,69 \%$, y está presente especialmente en la micro y pequeña empresa.

- En segundo lugar se posicionan las lógicas interorganizacionales, fuentes especializadas y TIC, con el $11,63 \%$, y se presentan en los tres tamaños analizados.

8 Nos referimos a la ponencia en el VIII Congreso Internacional de Análisis Organizacional Individuo y sociedad: Redes y formas emergentes de organización, en la Ciudad de Monterrey, Nuevo León, con el tema de Límites y posibilidades en las formas de aprendizaje en el sector manufacturero en las Mipymes. Caso de Celaya Guanajuato. 
- En tercer lugar se ubica la ausencia de información y conocimiento, con el 7,60\%. Los casos se presentan principalmente en la microempresa, $y$, en segundo término, en la pequeña empresa.

- En cuarto lugar están las denominadas otras formas culturales de información, con 3,50\%, principalmente en la pequeña empresa, seguido por la microempresa, y no se presentan casos en la mediana empresa.

- En quinto lugar se ubican las fuentes organizacionales, con $3,10 \%$, destacando la mediana, posteriormente la pequeña y por último la microempresa.

- En sexto lugar están las fuentes formales de conocimiento, con 3,02\%, estando presentes en los tres tamaños, principalmente en la mediana, le sigue la pequeña y después la microempresa.

- Los medios de comunicación y publicidad representan el 1,95\%, y están presentes solamente en la micro y en la pequeña empresa.

\section{A manera de conclusión}

El marco de la interpretación es la cultura, en donde el conocimiento y el aprendizaje se dan en la relación código - decodificación en distintas modalidades de la información. Esto implica que existe una capacidad (capital cultural) de un sujeto para interpretar la información cuando la necesita, y a esa apropiación simbólica la denominamos aprendizaje. El aprendizaje para contenidos conceptuales es lo que se denomina conocimiento (puede haber aprendizaje de habilidades o aprendizaje no cognitivo como los valores).

Las fuentes culturales de información para el aprendizaje, consultadas por las empresas, pueden estudiarse desde diversas aristas. Los enfoques existentes han privilegiado una valoración cuantitativa con respecto a su importancia. Sin menoscabar la influencia sectorial y por tamaño, al parecer se puede afirmar que existen ciertas regularidades que prevalecen para todo tipo de industrias, como pueden ser la alta valoración que se les da a los clientes y proveedores como fuentes externas de información, así como a la propia experiencia productiva, expresada en las áreas funcionales como el departamento de producción y mercadotecnia o en el expertise de grupos de trabajadores, gerentes o dueños. Para los países en desarrollo y, en particular, para la región latinoamericana, la información catalogada o codificada en revistas, libros o catálogos puede ser muy trascendente, además de la utilización del internet.

Desde la perspectiva del enfoque cualitativo de análisis existen múltiples posibilidades aún inexploradas. Construir un marco de análisis constituye un reto, dadas las múltiples categorías relevantes como pueden ser la confianza, adecuación, complejidad, complementariedad o consistencia de las fuentes, además de la naturaleza dual del conocimiento, tácito y codificado, junto con su proceso de generación, apropiación y uso, tal como se manifiesta en la fuente de aprendizaje de familia, que no había sido considerada como una fuente importante entre las organizaciones comerciales, sobre todo en las pequeñas y medianas. Nuestra apuesta ha sido por una valoración basada en el propósito de las fuentes, así como en el uso adecuado de los recursos de información.

A partir de la configuración y valoración de las fuentes de información que utilizan las Mipymes en Celaya para aprender sobre su actividad, ¿qué se puede decir de los sistemas de negocio? Son sistemas diversos pues los empresarios identificaron 26 modos diferentes de proveerse de información para aprender. Sin embargo el uso de estas fuentes no privilegia los canales interactivos sino los canales de compra o transacción, lo cual no favorece ni la generación de derramas ni el desarrollo de las capacidades de absorción. Las bases de conocimiento se pueden sintetizar en el uso de información de fuentes pasivas especializadas, utilizando conocimiento de la propia experiencia, de fuentes de conocimiento institucionalizadas, además del uso de otras fuentes culturales, y facultando sus soluciones en las fuentes organizacionales y la práctica inmediata autorreferencial.

Aun en el estrato de micro, pequeñas y medianas empresas podemos encontrar diferencias que se expresan en los mecanismos formales de aprendizaje, las fuentes organizacionales y los socios de la vinculación.

Los resultados contribuyen a conocer las fuentes de información y conocimiento de las empresas de Celaya, y, con base en ello, potenciar los vínculos con fuentes organizacionales y consolidar dichos vínculos con relaciones de confianza e integración a través de programas y políticas específicas, apoyadas en instituciones educativas que mejoren la capacidad de aprender. Sin embargo, 
la evidencia apunta a que el gobierno está ausente, falla en la señalización de los incentivos de sus programas o no difunde con eficacia sus objetivos.

\section{Referencias}

Aitken, B., Hanson, G. H. \& Harrison, A. E. (1997). Spillovers, Foreign Investment, and Export Behavior. Journal of International Economics, 43, 103-132.

Anlló, G. \& Suárez, D. (2008). Innovación: algo más que $I+D$. Evidencias Iberoamericanas a partir de las encuestas de innovación: construyendo las estrategias empresarias competitivas en El Estado de la Ciencia 2008, Buenos Aires: RICYT.

Arvanitis, R. \& Villavicencio, D. (1998). Technological Learning and Innovation in the Mexican Chemical Industry: An Exercise in Taxonomy. Science, Technology and Society, 3(1), 153-180.

Arthur, W. B. (1989). Competing Technologies, Increasing Returns, and Lock-In by Historical Events. Economic Journal, 99, 116-131.

Barañano, A. M. (1998). A Relação entre a Inovação e a Dimensão de Empresas. XX Simpósio de Gestão da Inovação Tecnológica, São Paulo.

Blomström, M. \& Kokko, A. (1998). Multinational Corporations and Spillovers. Journal of Economic Surveys, 12(3), 247-277.

CIPI. (2003). Observatorio de la pequeña y mediana empresa. Encuesta de la pequeña y mediana empresa 2002. Comisión Intersecretarial de Política Industrial - Secretaría de Economía, México.

Coecytjal (2003) Programa Estatal de Ciencia y Tecnología de Jalisco. Guadalajara, Gobierno del Estado de Jalisco.

Cohen, W. \& Levinthal, D. (1990). Absorptive Capacity: A New Perspective on Learning and Innovation. Administrative Science Quarterly, 35(1), 128-152.

Conacyt (1997) Encuesta Nacional de Innovación (ENI) 1996. México, DF: Consejo Nacional de Ciencia y Tecnología.

Conacyt. (1999, 2003). Encuestas Nacionales de Innovación en el sector manufacturero. Consejo Nacional de Ciencia y Tecnología. México.

Corona Alcántar, J. M. \& Hernández, C. (2000, septiembre). Relación proveedor-usuario y flujos de información tecnológica en la industria mexicana. Comercio Exterior, Bancomext, 50(9).

Daim, T. \& Kocaoglu, D. F. (1998, February 16-20). Technology Acquisition in the US Electronics Manufacturing Industry. Management of Technology, Sustainable Development and Eco-Efficiency. Seventh International Conference on the Management of Technology held in Orlando, Florida.

DICYT (2006). La Innovación en la Industria Uruguaya (2001-2003). II Encuestas de Actividades de
Innovación en la Industria. Montevideo: Dirección de Innovación, Ciencia y Tecnología para el Desarrollo.

Dosi, G. (1982). Technological Paradigms and Technological Trajectories: A Suggested Interpretation of the Determinants and Directions of Technical Change. Research Policy, 11(3), 147-162.

Dosi, G. (1988). The Research on Innovation Diffusion: an Assesment. En Nakicenovic, N. y A. Grubler, (Eds.). Diffusion of Technologies and Social Behavior (pp. 179-208). Berlin: Springer-Verlag.

Dosi, G. (1997). Opportunities, Incentives and the Collective Patterns of Technical Change. Economic Journal, 107, 1530-1547.

Drouvot, H. \& Fensterseifer, J. E. (2002). O papel das redes de cooperação nas políticas de inovação tecnológica das pequenas e médias empresas. Encontro anual da Nacional da Associação de Programas de Pós-Graduação em Administração, 2002, Anais Eletrônicos, Bahia, 2002, CD-ROM.

Dutrénit, G. \& Vera-Cruz, A. (2000, septiembre) Fuentes de conocimiento para la innovación en la industria química mexicana. Revista Comercio Exterior, 50(9), 786-798.

Dutrénit, G. \& De Fuentes, C. (2009). Derramas de conocimiento y capacidades de absorción. En Dutrénit, G. (Ed.). Sistemas regionales de innovación: un espacio para el desarrollo de las Pymes. El caso de la industria de maquinados industriales (pp. 3354). Colonia Suiza: UAM/Textual S.A.

Estrada, S. (2005). Economía de la innovación en el contexto mexicano. Ponencia especialmente preparada para el Programa Semanal de Seminarios de la Escuela de Economía. Universidad de Guanajuato. Marzo 11. Recuperado de http://www.ingenierias. ugto.mx/profesores/salvadorer/documentos/IDTcap $\% 201$ exp_prof_Economia $\% 20 \mathrm{de} \% 20 \mathrm{la} \% 20$ innovacion.doc.

Estrada, S. (2006, enero-abril). Diferencias regionales en la conducta tecnológica de las empresas manufactureras mexicanas: el caso de Guanajuato. Revista Economía, Sociedad y Territorio, V (20), 821-869.

Estrada, S., Cárcamo, L. \& Martín, M.L. (2013, Julio-Diciembre) Economía de los servicios. Revista GIS. Campus Celaya-Salvatierra, 2, 185-200.

Eurostat (2008) Science, Technology and Innovation in Europe (2008 edition) Luxembourg: Eurostat.

Foss, N. J. (1999). Perspectives on Business. International Studies of Management \& Organization, 29.

Garza, G. (2009, 26-28 de agosto). La revolución macroeconómica del sector servicios: Estados Unidos, México, Nuevo León y Guanajuato. Mesa de Trabajo 3: Economía de los servicios, industrias creativas e intermediación. 4o. Congreso Internacional Sistemas de Innovación para la Competitividad 
2009. Consejo de Ciencia y Tecnología del Estado de Guanajuato. Universidad Iberoamericana, León. Hernández, C. A. (1998). La relación innovativa entre el productor y el usuario de tecnología: el papel del usuario en el sector de aparatos de medición y control. Tesis de maestría. Universidad Autónoma Metropolitana-Xochimilco, México.

Hillmann, K-H. (2001). Diccionario Enciclopédico de Sociología. España: Herder.

IBGE. (2007). Pesquisa de Inovação Tecnológica- PINTEC 2005. Rio de Janeiro: Instituto Brasileiro de Geografia e Estatística.

Indec (2006) Encuesta Nacional a empresas sobre Innovación, I+D y TICS [2002-2004]. Buenos Aires: Instituto Nacional de Estadística y Censos.

INE (2008) Encuesta sobre innovación tecnológica de las empresas 2006. Madrid: Instituto Nacional de Estadística.

Inegi (2007) Módulo de Innovación Tecnológica 2008, Aguascalientes: Instituto Nacional de Estadística, Geografía e Informática.

Inegi. (2010). Censos Económicos 2009 en Guanajuato. Recuperado de http://www.inegi.org.mx/est/ contenidos/espanol/proyectos/censos/ce2009/ calc_CENSAL-municipio.asp

López-Leyva, S. (2003). Empresarios e innovación tecnológica en Sinaloa. Región y Sociedad, XV(27).

Lugones, G., Gutti, P. \& Le Clech, N. (2007). Indicadores de capacidades tecnológicas en América Latina. Serie Estudios y Perspectivas $n .^{\circ} 89$. Sede Subregional de la Cepal en México. Series Cepal, Unidad de Comercio Internacional e Industria. Canada International Development Agencia. Naciones Unidas, México, DF. 68 pp.

Lundvall, B.-A., (Ed.). (1992). National Systems of Innovation: Towards a Theory of Innovation and Interactive Learning. London: Pinter Publishers.

OCDE. (2000). A New Economy? The Changing Role of Innovation and Information Technology in Growth. Paris: OECD Publishing,
OCDE. (2005). Oslo Manual: Guidelines for Collecting and Interpreting Innovation Data (3rd. ed.). Paris: OECD Publishing.

OCYT (2004). Segunda Encuesta de Desarrollo Tecnológico (EDT2). Bogotá: Observatorio Colombiano de Ciencia y Tecnología.

Ollivier Fierro, J. O. \& Thompson Gutiérrez, P. I. (2009, enero-abril). Diferencias en el proceso de innovación en empresas pequeñas y medianas de la industria manufacturera de la ciudad de Chihuahua, México. Revista Contaduría, 227, 9-28.

Pastor, M.P. \& Medina, R. (2008). Sistema de innovación en San Luis Potosí: innovación en la industria metalmecánica. III Congreso Internacionl de Sistemas de Innovación para la Competitividad. Guanajuato: Consejo de Ciencia y Tecnología del Estado de Guanajuato.

Porto, G. Oliveira, F. \& Ary, G. (2003, octubre 22-24). As fontes de tecnologia no setor de telecomunicações e os fatores motivadores para cooperação. Ponencia presentada en el X Seminario Latino-Iberoamericano de Gestión Tecnológica Altec: conocimiento, innovación y competitividad: los desafíos de la globalización, México D. F., México.

Quadros, R., Furtado, A., Bernardes, R. \& Franco, E. (2001). Technological Innovation in Brazilian Industry: An Assessment Based on the São Paulo Innovation Survey. International Journal of Technological Forecasting and Social Change, 67(2).

Romer, P. (1990, October). Endogenous Technological Change. Journal of Political Economy, 98(5), S71102.

Unger, K. (2009). Competitividad y especialización de la economía de Guanajuato: un acercamiento municipal, 1993-2000. Documento de Trabajo del CIDE, 462. México, DF.

Whitley, R. (1992). Business Systems in East Asia: Firms, Markets, and Societies. London: Sage. 280 pp. 


\section{ANEXO I. Revisión de la literatura sobre fuentes de información y conocimiento}

\begin{tabular}{|c|c|c|c|c|c|c|c|c|c|c|c|c|c|}
\hline AUTORES & 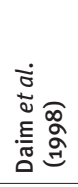 & 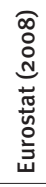 & 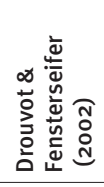 & 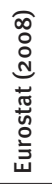 & 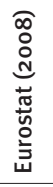 & 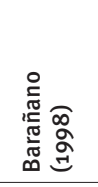 & 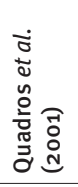 & 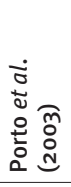 & 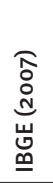 & 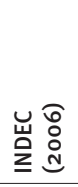 & 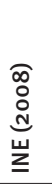 & 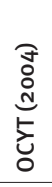 & 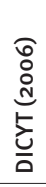 \\
\hline REGIÓN/PAÍS & $\Xi$ & $\stackrel{山}{\ll}$ & 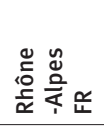 & 똔 & जे & 占 & $\begin{array}{l}\frac{0}{\overline{7}} \\
\frac{0}{\circ} \\
\text { 芯㠃 }\end{array}$ & $\stackrel{\circ}{\infty}$ & $\stackrel{\circ}{\infty}$ & 产 & $\overline{\mathbf{I}}$ & ठํ & 总 \\
\hline SECTOR & 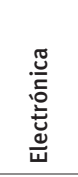 & & 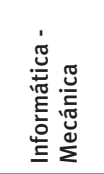 & & & 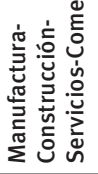 & 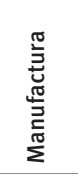 & 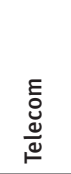 & & & & & \\
\hline TAMAÑO & & & Pymes & & & Todos & & & & & & & \\
\hline Otras empresas & & + & & + & & + & & & & & + & & \\
\hline Revistas especializadas & + & & & & & + & & & & & & & \\
\hline \multicolumn{14}{|l|}{ Libros } \\
\hline Ferias y exposiciones & + & & & & & + & & + & & & & & \\
\hline Competidores & & & & & & + & + & & + & & & & \\
\hline Grupos internos & & + & & + & + & + & & + & + & + & + & & + \\
\hline Proveedores & + & + & + & + & + & + & + & & + & + & + & + & + \\
\hline Clientes & & + & + & + & + & + & + & + & + & + & + & & + \\
\hline Univ. y centros I+D & & + & & & + & + & & + & & & & & \\
\hline Gobierno & & & - & & & & & & & & & & \\
\hline Capacitación & + & & & & & & & & & & & + & \\
\hline Conferencias y simposios & & & & & & & & + & & & & & \\
\hline \multicolumn{14}{|l|}{ Internet } \\
\hline Consultores & & & & & + & & & & & + & & & + \\
\hline \multicolumn{14}{|l|}{ Patentes } \\
\hline Diferencia pymes & & & & & & $x x$ & $x X$ & & & & & & \\
\hline
\end{tabular}

Donde (+) indica la importancia en la percepción o la preponderancia en la frecuencia de respuesta y (XX) indica que los estudios encuentran diferencias significativas. 


\begin{tabular}{|c|c|c|c|c|c|c|c|c|c|}
\hline AUTORES & 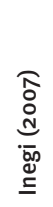 & 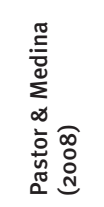 & 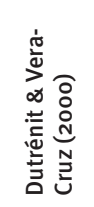 & 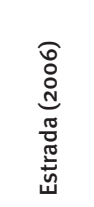 & 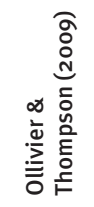 & 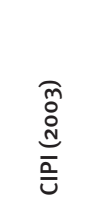 & 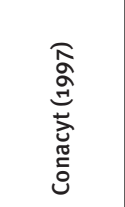 & 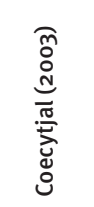 & 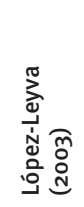 \\
\hline REGIÓN/PAÍS & $\stackrel{x}{\Sigma}$ & 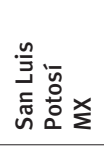 & $\stackrel{x}{\Sigma}$ & 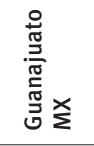 & 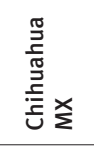 & $\stackrel{x}{\Sigma}$ & 出 $\stackrel{x}{\Sigma}$ & $\underline{\underline{0}} \stackrel{x}{\Sigma}$ & $i=\frac{x}{\Sigma}$ \\
\hline SECTOR & & 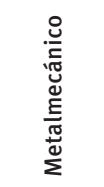 & 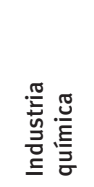 & 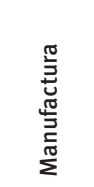 & 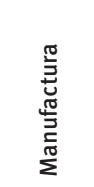 & 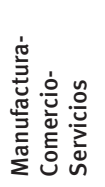 & 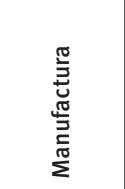 & 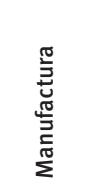 & 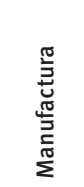 \\
\hline TAMAÑO & & Todos & Todos & Todos & Pymes & Pymes & $\begin{array}{l}\text { Med- } \\
\text { Grandes }\end{array}$ & Todos & Todos \\
\hline Otras empresas & + & & & & & & & & \\
\hline Revistas especializadas & & + & & & + & & & & \\
\hline Libros & & & & & + & & & & \\
\hline Ferias y exposiciones & & + & & + & & & + & & + \\
\hline Competidores & + & & + & & & & & + & \\
\hline Grupos internos & & + & + & + & & + & + & & \\
\hline Proveedores & + & + & & & + & + & + & + & \\
\hline Clientes & + & + & + & + & + & + & + & + & \\
\hline \multicolumn{10}{|l|}{ Univ. y centros I+D } \\
\hline \multicolumn{10}{|l|}{ Gobierno } \\
\hline \multicolumn{10}{|l|}{ Capacitación } \\
\hline Conferencias y simposios & & + & & & & & & & \\
\hline Internet & & & & & + & + & & & \\
\hline Consultores & & & + & & & + & & & + \\
\hline \multicolumn{10}{|l|}{ Patentes } \\
\hline Diferencia pymes & & & & & & & & & \\
\hline
\end{tabular}

Donde (+) indica la importancia en la percepción o la preponderancia en la frecuencia de respuesta y (XX) indica que los estudios encuentran diferencias significativas. Fuente: elaboración propia con base en Suárez \& Anlló (2008), Portos et al. (2003) y otras fuentes. 


\section{ANEXO II. Composición cultural de las fuentes de información y conocimiento}

\section{FUENTES DE INFORMACIÓN Y CONOCIMIENTO}

\section{LÓGICAS INTERORGANIZACIONALES, FUENTES ESPECIALIZADAS Y TIC}

Consulta a

instituciones

Las instituciones pueden trabajar con las empresas, no solo con la información disponible y pertinente sobre un problema, sino generando nuevos conocimientos por medio de la investigación, apoyadas además con equipos, laboratorios y una cadena de transmisión de conocimiento que puede tener un impacto interesante en la difusión del conocimiento de los casos de la región. Son fundamentales la relación y articulación con centros de educación superior e investigación con las empresas, sobre todo para el cambio hacia la innovación.

\section{Revistas}

Las revistas desempeñan un papel primordial, sobre todo las revistas especializadas en ciencia, tecnología de campos específicos o en campos empresariales, por ejemplo, gremios. Las revistas en México no son tan diversas y especializadas; su limitación está dada por la cobertura de su circulación. Las versiones electrónicas son ahora un elemento estratégico para la difusión del conocimiento.

Ferias

Las ferias y exposiciones: cabe aclarar que son dos tipos de ferias y exposiciones; por un lado están las ferias en las que solo se exhibe, y por otro, las ferias tecnológicas. Las dos son importantes, ya que en una se exponen los productos terminados, y eso permite ver el grado de avance de estos (a los mismos productores, obviamente, y a los consumidores), y eso mejora el nivel de práctica social de los productores, mientras en las ferias tecnológicas se hace énfasis en el desarrollo de las tecnologías. Pueden constituirse en los grandes detonadores para ampliar el panorama de los empresarios, ya que se presentan las novedades tecnológicas importantes, las mejoras de productos, las innovaciones. En México hay cierto potencial en el que se pueden complementary valorar (capital simbólico) esos espacios de aprendizaje.

Catálogos

Los catálogos o folletos tienen como fin brindar una información sintetizada de los productos (para vender), pero funcionan más como fichas técnicas en las que se destacan las características de los productos. Estos tienen una presencia física (en papel), y ahora se están diseñando catálogos en portales de internet.

Internet

Se habla de internet como un medio excelente que ha permitido dinamizar a la sociedad en cuanto a búsquedas de información; sin embargo, sabemos que la mayoría de las búsquedas son azarosas y ciegas, que aun en la práctica hay pocas búsquedas inteligentes y avanzadas, donde se pueda ir directamente por estas súper pistas a la información pertinente y llegar a buen puerto de los objetivos. El medio es importante cuando se apoya en herramientas como inteligencia competitiva.

FUENTES FORMALES DE CONOCIMIENTO

\begin{tabular}{ll}
\hline Talleres & El taller es una modalidad creativa de obtención de conocimiento y de transformación de \\
& habilidades; su premisa es conocer-haciendo o aprendiendo- transformando. Los talleres buscan la \\
& aplicación de un saber en una acción dada a la creación aplicada.
\end{tabular}

Asesorías

Es el conocimiento de un especialista que sugiere, de acuerdo con su experiencia y conocimiento técnico, un proceder de acción, en este caso de la empresa.

El asesor tiene la habilidad "didáctica" de describir los problemas complejos en palabras coloquiales. Es importante que se le evalúe sobre resultados, profesionalismo y dedicación. Tiene como ventaja cierta libertad, ya que no depende directamente de la empresa, y por tanto puede tener una opinión más sensata y no tan dependiente de las lógicas de poder, aunque finalmente es empleado del empresario.

Cursos

Los cursos tienen una orientación más cognitiva que las capacitaciones; los cursos son importantes ya que pueden dar las bases necesarias sobre algún conocimiento determinado, sobre todo aplicado.

Libros

Los libros han sido históricamente una fuente de saber fundamental; que implica para el intérprete una búsqueda de conocimiento (una cultura de la lectura); sin embargo, en nuestro país es escasa la práctica de la lectura (muy limitada por la deficiencia de comprensión, la falta de acceso y la falta de diversidad de temas actualizados). Sigue siendo una de las formas adecuadas sobre todo para personas con competencias lectoras y para autodidactas. 


\begin{abstract}
Seminarios
Los seminarios tienen como fin abordar temas de una manera completa y abierta; no son clases, ni tampoco talleres; implican partir de los conocimientos de los participantes, para profundizar de manera dirigida con el apoyo de lecturas o información pertinente.
\end{abstract}

Capacitaciones Las capacitaciones permiten facultarse en temas o tareas específicos, que tienen que ver más con las habilidades y destrezas; habilitarse, capacitarse, está más orientado a prácticas, y con ciertos conocimientos técnicos, esta es la orientación. No es para "conocer" de manera más profunda.

Estudios

Realizar estudios es una forma adecuada de informarse y de tener conocimientos. Es un proyecto más a mediano o largo plazo. Incrementar la capacidad en cuanto al capital cultural de los empresarios es importante; el problema manifiesto es el tiempo, aunque también es importante para los estudios la inversión en tiempo.

Conferencias

Las conferencias son otra fuente de información, no tan periódica en la cultura empresarial, pero es fundamental traer expertos en asuntos que son necesarios en cuestiones generales de temas desde una perspectiva tanto más científica o tecnológica, como específica. Sin embargo, es importante dejar en segundo término a los "motivadores" o "gurús" que son quienes han predominado en la escena de las conferencias.

\title{
FUENTES ORGANIZACIONALES
}

Experiencias de otras Está contemplada como una de las fuentes de conocimiento e información excelente (cuando empresas es sistematizada por ejemplo a través de inteligencia competitiva, en la que hay indicadores comparativos explícitamente señalados), pero, desde la mirada empirista del dueño, es interesante pero limitada, ya que no hay una evaluación rigurosa comparativa, solo un aprendizaje parcial en cuanto a la observación (no sistemática); se va a hablar de unos cuantos rasgos del producto, de los sistemas, de la asistencia, etc. También depende del nivel de la cobertura de comparación: si se hace con los mismos del gremio local, es muy limitada; si se amplía la cobertura a nivel regional, nacional y sobre todo internacional, puede ser más significativa.

Consulta a clientes De la misma manera, la consulta a clientes debe ser sistemática y detallada para que sea excelente (como sistema), así articulada a los procesos de seguimiento, a las sugerencias de los clientes, orientada a mejorar el producto o el servicio, no de manera informal como se lleva a cabo (generalmente), donde se le pregunta al cliente y este solo afirma o niega la impresión que tiene con referencia al producto o servicio, sin pretender innovar o mejorar.

Proveedores

Los proveedores potenciales y actuales tienen información necesaria para la toma de decisiones en cuanto a ciencia y tecnología; ellos sí pueden (y deben) dar especificaciones de su producto de manera muy detallada, asesorar en cuanto a requerimientos técnicos y demás. Deben demostrar hasta satisfacer la información de su producto (es conveniente que en esa diligencia se acompañe de un experto asesor por parte del empresario).

Consulta al gobierno El gobierno posee distintas secretarías y dependencias en los tres niveles de gobierno, que tienen la obligación de informar; se puede pedir asesoría en cuanto a un tema específico y dentro de su competencia en servicio de la sociedad.

Cámaras empresariales

Las cámaras empresariales como organismos intermedios están facultadas a impulsar y apoyar a sus agremiados; sin embargo, se presentan casos en los que la limitación es del representante, pero hay otras cámaras que sí han hecho un buen papel de asesoría y de gestión. Ampliar y dinamizar sus servicios pertinentes con los empresarios puede ser el mejor canal, ya que la representación de varias empresas posibilita disminuir costos y hacer sinergias.

Experiencia en otros
trabajos

Parte de la experiencia que los empresarios han tenido en otros trabajos, es una experiencia útil pero limitada, ya que su carácter práctico la obliga a resultados inmediatos sin que se dé un procesamiento o documentación de la experiencia o del conocimiento derivado. La experiencia en otros trabajos se adquiere por lo general de otros dueños, es decir, no es autorreferida.

Reuniones o juntas

Las reuniones o juntas dentro de la dinámica de autoaprendizaje de grupos de productores o de miembros de empresa son interesantes, pero se corre el riesgo de la autocomplacencia grupal, el reparto de roles de los participantes, la queja y la catarsis colectiva. Depende mucho de su dinámica. Es fundamental en la planeación democrática.

Certificaciones
Las certificaciones tienen como objetivo garantizar procesos en el sistema que está interconectado con diferentes acciones y variables relacionadas con las actividades principales o con el todo. Las certificaciones, como fuente cultural, constituyen una interpretación sobre el uso de esta fuente, no como un fin sino como un medio para proyectarse en el mercado. 


\section{MEDIOS DE COMUNICACIÓN Y PUBLICIDAD}

Televisión La televisión es un medio de comunicación, pero no una fuente de consulta confiable, ya que puede dar cierta información muy limitada (salvo en noticieros tecnológicos, programas especializados de ciencia o tecnología, documentales, sobre todo en televisión de cable o programación satelital), y en la televisión comercial su objetivo es el entretenimiento. Como fuente de consulta tendría que esperar a que se programaran temas de interés.

Periódicos

Los periódicos tienen distintas clases de información: noticias, publicidad, algunas secciones de ciencia y tecnología. La información es limitada (excepto cuando hay reportajes). En la actualidad no se va a dar información detallada, ya que por la tendencia de los medios hacia la construcción social del evento se recurre al sensacionalismo; no se publica información detallada de ciencia o tecnología porque el público se puede aburrir (y esta información se considera que no es rentable).

Publicidad La publicidad está orientada a vender; puede tener información importante en cuanto a ciertas características enunciadas (sin mucha especificación), pero no va a dar detalles, sobre todo cuando hay información que podría ser adversa a su objetivo de vender. La persuasión, como un instrumento para vender, puede hacer que se exageren las características o propiedades de lo referido.

OTRAS FORMAS CULTURALES DE INFORMACIÓN

Familiares

La experiencia que han tenido parientes en una actividad económica similar es parte relevante del capital social en la creación y el mantenimiento de empresas. Es una fuente valiosa del saber popular en cuanto a capital cultural de apoyo de información y de conocimiento, pero tiene limitantes relacionadas con la arbitrariedad del sujeto consultado y su conocimiento real de las cosas referidas. Sin embargo, es un medio que no se debe desdeñar.

\section{NO RELACIÓN CON OTRAS FUENTES}

Experiencia directa La experiencia directa es una forma de conocimiento denominado empírico; el problema es que es autorreferido. Como saber práctico es funcional, pero no profundiza porque ese no es su fin. Queda a nivel implícito (para quien lo posee) y otras veces se transmite con niveles amplios de doxa y arbitrio.

\section{AUSENCIA DE INFORMACIÓN Y CONOCIMIENTO}

No sabe Manifiesta explícitamente que no sabe de ninguna fuente para consultar en cuanto a la información necesaria para la producción y la innovación.

Ninguna Ausencia de respuesta.

Fuente: elaboración propia con base en Suárez \& Anlló (2008), Portos et al. (2003) y otras fuentes. 